\title{
LOS ACADÉMICOS FRENTE A LA GESTIÓN DE LA CALIDAD EN LAS INSTITUCIONES DE EDUCACIÓN SUPERIOR. PERCEPCIÓN Y VALORACIÓN
}

\author{
Jon Olaskoaga LaRRauri* \\ Elia Marúm Espinosa** \\ Víctor Manuel Rosario Muñoz*** \\ DAVID PÉREZ LECHOSA****
}

\begin{abstract}
* Doctor en Economía por la Universidad del País Vasco/Euskal Herriko Unibertsitatea. Profesor titular en la Escuela de Estudios Empresariales de dicha Universidad. Euskadi, España. E-mail: jon.olaskoaga@ehu.es ** Doctora en Economía de la Universidad Nacional Autónoma de México. Profesora investigadora y Directora del Centro para la Calidad e Innovación de la Educación Superior. Universidad de Guadalajara. Zapopan, México. E-mail: emarum@cencar.udg.mx

*** Doctor en Educación por la Universidad de Guadalajara. Profesor-investigador en el Centro Universitario de Ciencias Económico Administrativas en el Centro para la Calidad e Innovación de la Educación Superior en la Universidad de Guadalajara. Trabaja el tema de la gestión universitaria y desarrolla la línea de investigación curriculum, práctica docente y cambio institucional en la educación superior. Participante en proyectos de investigación internacional sobre políticas de calidad en la Región Iberoamericana. Creador de la Red Retos y Expectativas de la Universidad (2000) y miembro fundador y creador de la idea de la Red de Académicos de Iberoamérica A.C. (2011). E-mail: vrosario14al18@hotmail.com

Graduado en Gestión de Negocios por la Universidad del País Vasco (UPV/EHU). Asesor financiero. Bilbao, España. E-mail: dperezlechosa@hotmail.com.
\end{abstract}

Resumen: Este artículo reúne y ordena la evidencia empírica existente sobre las opiniones y actitudes de los académicos universitarios frente a la introducción, en sus instituciones, de la lógica, los principios y las herramientas de la gestión de la calidad. El enfoque adoptado es el de la meta-investigación, que supone la recopilación de la literatura relevante y la sistematización de sus contenidos. Éstos se han expuesto ordenados en tres líneas temáticas: las nociones de calidad aceptadas por los académicos, su percepción de los logros de la gestión de la calidad, y su valoración de los diferentes métodos de evaluación asociados a los sistemas de garantía de la calidad.

Palabras clave: Gestión de la calidad educativa. Calidad de la educación superior. Académicos y gestión institucional de la calidad. Percepciones de la calidad académica

\section{FACULTY FACING QUALITY MANAGEMENT IN HIGHER EDUCATION INSTITUTIONS. PERCEPTION AND ASSESSMENT}

Abstract: This paper puts together and arranges the existing empirical evidence on opinions and attitudes of faculty members about the introduction in their institutions of the logic, principles and tools of quality management. The approach used was the meta-research, which involves compilation of the relevant literature and systematization of its topics. These have been exposed into three thematic lines: the notions of quality accepted by faculty members, their perception of the achievements of quality management, and their assessment of the different evaluation methods associated with systems of quality assurance.

Key words: Management of education quality. Quality of higher education. Faculty and institutional quality management; Perceptions of quality.

OS ACADÊMICOS FRENTE À GESTÃO DA QUALIDADE NAS INSTITUIÇÕES DE EDUCAÇÃO SUPERIOR. PERCEPÇÃO E VALORAÇÃO 
Resumo: Este artigo reúne e ordena a evidência empírica existente sobre as opiniões e atitudes dos acadêmicos universitários diante da introdução, em suas instituições, da lógica, princípios e ferramentas da gestão da qualidade. O enfoque adotado é o da metainvestigação, que supõe a recopilação da literatura relevante e a sistematização de seus conteúdos. Estes foram expostos em três linhas temáticas: as noções de qualidade aceitas pelos acadêmicos, sua percepção dos sucessos da gestão da qualidade e sua valoração dos diferentes métodos de avaliação associados aos sistemas de garantia da qualidade.

Palavras-chave: Gestão da qualidade educativa. Qualidade da educação superior. Acadêmicos e gestão institucional da qualidade. Percepções da qualidade acadêmica.

\section{INTRODUCCIÓN}

Puede que las instituciones universitarias sean poco permeables a las tendencias que surgen y se extienden en su entorno. Sin embargo, la historia reciente demuestra que hay ocasiones en que no pueden sustraerse a lo que se mueve a su alrededor. Y así, en los noventa, son apreciables dos fenómenos. Por un lado, la acumulación de una doctrina favorable a la aplicación en la universidad de los objetivos, los principios y las herramientas de la gestión de la calidad. Por otro, la puesta en marcha de políticas de evaluación que propiciaron, cuando no exigieron, un giro hacia la gestión de la calidad en las instituciones universitarias.

Sin embargo, los términos en los que la gestión de la calidad ha comenzado a ensayarse en las instituciones universitarias todo el mundo han generado dudas entre los académicos. Algunos han descubierto en la gestión de la calidad una ocasión inmejorable para poner en marcha cambios y reformas largo tiempo demandadas. Otros, en cambio, opinan que las nuevas políticas conculcan principios tradicionales que se encuentran en la base del funcionamiento de las instituciones universitarias.

Este artículo se ha escrito con la intención de reunir y ordenar la evidencia empírica existente sobre las opiniones y actitudes de los académicos frente a la introducción, en sus instituciones, de la lógica, los principios y las herramientas de la gestión de la calidad.

\section{BREVE DIGRESIÓN METODOLÓGICA}

Este artículo contiene una meta-investigación sobre la opiniones y las actitudes de los académicos frente a la gestión de la calidad, un campo en el que la evidencia se encuentra muy fragmentada y en el que esta propuesta metodológica puede cumplir la función de ordenar y sistematizar la evidencia existente. En la determinación del material bibliográfico relevante se emplearon los siguientes criterios: los trabajos seleccionados deberían: 
i. Tener una orientación eminentemente empírica.

ii. Recoger opiniones de personal académico en instituciones de educación superior (IES).

iii. Tratar la cuestión de la gestión de la calidad en IES u otros temas concomitantes como, por ejemplo, los procesos de integración de sistemas universitarios nacionales y sus consecuencias.

La búsqueda de materiales se llevó a cabo desde la experiencia de los autores del artículo en esta línea de investigación. También se realizó una búsqueda más sistemática en trece revistas de reconocido prestigio $^{1}$ y a través de las herramientas de búsqueda de los índices ISI, JSTOR y SCOPUS ${ }^{2}$

Los resultados de las búsquedas se analizaron uno por uno, siguiendo los requisitos establecidos, dando como resultado un total de 22 artículos cuya caracterización se aborda en la tabla 1.

\section{Pensamientos, percepciones y opiniones de los docentes uni- versitarios a propósito de la gestión de la calidad}

Este epígrafe reúne y sistematiza la evidencia acumulada en el campo de las opiniones y actitudes de los académicos sobre la gestión de la calidad en la enseñanza universitaria. Por conveniencia el relato se divide en tres partes relativas a:

i) El significado de la calidad de la enseñanza para los académicos.

ii) Su valoración de la introducción de prácticas e instrumentos de gestión de la calidad en la enseñanza universitaria.

iii) Sus opiniones sobre sistemas de evaluación.

\section{El significado de la calidad de la enseñanza para los académicos}

Dado que no existe consenso sobre el significado de "calidad" en general (BOADEN, 1997) tampoco debe extrañar que no se haya alcanzado en el terreno menos pacífico de la enseñanza universitaria. En este ámbito conviven hasta cinco maneras diferentes de entender la calidad Harvey y Green (1993):

1 Las siguientes revistas se revisaron exhaustivamente: Archivos Analíticos de Políticas Educativas, Assessment \& Evaluation in Higher Education, Educational Policy, Higher Education, Higher Education, International Journal of Educational Management, International Journal of Higher Education, International Journal of Higher Education, International Journal of Quality \& Reliability Management, Quality Assurance in Education, Quality in Higher Education, REICE - Revista Electrónica Iberoamericana sobre Calidad, Eficacia y Cambio en Educación, Review of Higher Education, Teaching in Higher Education y The TQM Magazine.

2 Se emplearon los siguientes términos de búsqueda y sus correspondientes equivalentes en inglés: Calidad, ECTS, EEES, Encuesta, Opiniones de docentes / académicos, Plan Bolonia, TQM. 
Tabla 1- Literatura empírica sobre actitudes de los académicos.

\begin{tabular}{|c|c|c|}
\hline Trabajo & $\begin{array}{l}\text { Ámbito geográfico, } \\
\text { temporal e institucional }\end{array}$ & Agentes \\
\hline Elmuti et al (1996) & $\begin{array}{l}\text { Universidades del medio oeste de } \\
\text { los Estados Unidos, } 1995\end{array}$ & $\begin{array}{l}\text { Presidentes, decanos, jefes } \\
\text { de departamento, docentes, } \\
\text { staff de apoyo, estudiantes, } \\
\text { padres y ex alumnos }\end{array}$ \\
\hline Mclnnis (1998) & $\begin{array}{l}\text { Australia, 1993/94 (académicos); } \\
1996 \text { (administradores) }\end{array}$ & $\begin{array}{l}\text { Administradores y } \\
\text { académicos }\end{array}$ \\
\hline $\begin{array}{l}\text { Newton (1999); } \\
\text { Newton (2001) }\end{array}$ & $\begin{array}{l}\text { Un Centro Universitario del Reino } \\
\text { Unido. 1993-98 }\end{array}$ & $\begin{array}{l}\text { Administradores y } \\
\text { académicos }\end{array}$ \\
\hline Lomas (2002) & $\begin{array}{l}\text { Reino Unido. } \\
\text { 1999-2000 }\end{array}$ & Administradores \\
\hline Ferrer et al (2004) & $\begin{array}{l}\text { España (Madrid, País Vasco, } \\
\text { Valencia y Sevilla); } 2004\end{array}$ & Académicos \\
\hline $\begin{array}{l}\text { Watty } \\
\text { (2005); } \\
\text { Watty (2006) }\end{array}$ & $\begin{array}{l}\text { Enseñanzas de contabilidad. } \\
\text { Australia, } 2003\end{array}$ & Académicos de contabilidad \\
\hline Rosa et al (2006) & Portugal, 2005 & $\begin{array}{l}\text { Rectores y académicos } \\
\text { responsables de los informes } \\
\text { de auto evaluación }\end{array}$ \\
\hline Lomas (2007) & Reino Unido & Docentes \\
\hline Ursin et al (2008) & Finlandia (2004/05) e Italia (2006). & $\begin{array}{l}\text { Docentes y personal } \\
\text { universitario }\end{array}$ \\
\hline Cartwright (2007) & $\begin{array}{l}\text { Académicos de la universidad } \\
\text { británica trabajando después de la } \\
\text { reforma de } 1992 \text {. }\end{array}$ & $\begin{array}{l}\text { Académicos con y sin } \\
\text { responsabilidades de gestión } \\
\text { de la calidad. }\end{array}$ \\
\hline $\begin{array}{l}\text { Olaskoaga, J. (Coord.) } \\
\text { (2009); Cardona et al } \\
\text { (2009) }\end{array}$ & $\begin{array}{l}\text { Docentes de las áreas de economía } \\
\text { y empresa en Argentina (Persoglia; } \\
\text { López Armengol; Zoloa, 2009), } \\
\text { Chile (Araya; Arias; Ibarra, 2009), } \\
\text { España (Barrenetxea et al, 2009) y } \\
\text { México (Marúm et al, 2009), 2008. }\end{array}$ & Docentes \\
\hline García-García et al (2009) & $\begin{array}{l}\text { Profesores universitarios del área } \\
\text { de ingeniería en Italia (2006) y } \\
\text { España (2008) }\end{array}$ & Docentes \\
\hline Veiga y Amaral (2009) & $\begin{array}{l}\text { Universidades, institutos y escuelas } \\
\text { politécnicas de Portugal, } 2006\end{array}$ & $\begin{array}{l}\text { Rectores y coordinadores de } \\
\text { programas universitarios }\end{array}$ \\
\hline Fernández et al (2010) & $\begin{array}{l}\text { Universidad Complutense de } \\
\text { Madrid, Curso 2007/08 }\end{array}$ & Docentes \\
\hline Mijangos et al (2011) & $\begin{array}{l}\text { Universidades públicas españolas } \\
\text { que ofrecen enseñanza presencial. } \\
\text { España, } 2009 / 10\end{array}$ & Docentes \\
\hline $\begin{array}{l}\text { López Armengol y } \\
\text { Colombo (Coords.) (2011); }\end{array}$ & $\begin{array}{l}\text { Decanos y directores de Centros } \\
\text { Universitarios en el área de las } \\
\text { ciencias sociales y jurídicas, } 2009 .\end{array}$ & Administradores \\
\hline Kleijnen et al (2011) & $\begin{array}{l}\text { Holanda, } 2008 \\
\text { (Universities of Applied Science) }\end{array}$ & Académicos \\
\hline Cardona (Coord.) (2012) & $\begin{array}{l}\text { Universidades públicas con } \\
\text { enseñanza presencial en Argentina } \\
\text { (López Armengol et al, 2011), } \\
\text { España (Mijangos et al, 2011b) } \\
\text { y México, 2009/10 (Marúm et al, } \\
\text { 2011) }\end{array}$ & Docentes \\
\hline Vieira (2002) & $\begin{array}{l}\text { Universidad de Minho, Portugal, } \\
2000 / 01\end{array}$ & Docentes y estudiantes \\
\hline
\end{tabular}

Fuente: Elaboración propia 
- La calidad como algo excepcional es la forma tradicional de entender la calidad en la universidad. Hay unas pocas instituciones universitarias que consiguen sobresalir sobre las demás por la vía de disponer de los mejores docentes, los mejores estudiantes, las mejores instalaciones y, en general, lo mejores medios.

- La calidad como la perfección o la consistencia de los resultados de los procesos. Desde este punto de vista, la calidad consiste en reducir la variabilidad de los resultados de cualesquiera procesos que se lleven a cabo en una organización. En el contexto educativo es más difícil encontrar procesos repetitivos y, por tanto, el propio concepto pierde aplicabilidad.

- La calidad como adecuación una finalidad. Esta forma de entender la calidad resulta muy intuitiva; al fin y al cabo, la calidad es un atributo subjetivo de los productos y los servicios, que debería ser definida por quienes los adquieren y utilizan. La calidad de la enseñanza podría entenderse en función de su capacidad para satisfacer las expectativas de los estudiantes, pero también las de sus empleadores, o incluso las de otros agentes políticos y sociales.

- La calidad como eficiencia en la utilización de recursos ${ }^{3}$ es una idea tradicional de la doctrina administrativa. Toda gestión debe asegurar que los resultados se alcanzan sin un excesivo consumo de recursos. Se trata de una idea que se ha recuperado a partir de la extensión de la nueva administración pública y su defensa del principio de que toda iniciativa que reciba y utilice recursos públicos debe rendir cuentas por el empleo que hace de ellos.

- La calidad como transformación del estudiante. Es un concepto acuñado por Harvey y Green que encaja a la perfección con los principios de la pedagogía moderna. La enseñanza, en particular la enseñanza superior, tiene como propósito último convertir al estudiante en dueño de su propio destino.

La cuestión radica en con cuál de estas nociones se identifican más los académicos, admitido que aunque no existe incompatibilidad entre ellas, cada una representa una sensibilidad diferente con respecto a la institución universitaria y su función social.

Watty $(2006,2005)$ sometió directamente los conceptos de Harvey y Green (1993) a la consideración de los docentes en el área de conocimiento de contabilidad en una serie de universidades australianas, y se encontró con que la

3 En ocasiones se emplea "valor por dinero", por influencia de la expresión inglesa "value for money". 
noción preferida por un mayor porcentaje de docentes (77\% de respuestas "de acuerdo" o "muy de acuerdo" en una escala típica de cinco categorías) fue la que asocia calidad y transformación del estudiante.

Lo mismo ocurrió entre los docentes en el área de economía de las universidades de Argentina, Chile y España (OLASKOAGA, 2009) -el caso de México representa una excepción en este sentido-, y entre los docentes universitarios, con independencia de su área de conocimiento, en España (MIJANGOS et al, 2011), aunque en estos casos, las nociones de Harvey y Green fueron operacionalizadas de un modo diferente (ver tabla 2).

Los resultados de todos estos trabajos coinciden también en identificar un orden de prelación entre conceptos. Los docentes prefieren en primer lugar los conceptos ligados a la transformación; en segundo lugar están los relacionados con la adecuación a alguna finalidad; y sólo al final se encuentran los estándares académicos y a la eficiencia en el empleo de los recursos (tabla 2).

Tabla 2 - Identificación del profesor con diferentes conceptos de calidad. Porcentaje de respuestas "de acuerdo" y "muy de acuerdo".

\begin{tabular}{|c|c|c|c|c|}
\hline \multirow[b]{2}{*}{ Transformación } & & España & México & Argentina \\
\hline & $\begin{array}{l}\text { C1. La calidad consiste en desarrollar } \\
\text { las capacidades del estudiante para } \\
\text { avanzar en su propia transformación. } \\
\text { C2. La calidad consiste en formar } \\
\text { estudiantes capaces de asumir un } \\
\text { compromiso social. }\end{array}$ & $80 \%$ & $86 \%$ & $84 \%$ \\
\hline \multirow{3}{*}{$\begin{array}{c}\text { Adecuación a } \\
\text { un fin }\end{array}$} & $\begin{array}{l}\text { C4. La calidad consiste en satisfacer las } \\
\text { expectativas de todos los involucrados } \\
\text { (alumnos, profesores, instituciones, } \\
\text { sociedad). }\end{array}$ & $61 \%$ & $74 \%$ & $61 \%$ \\
\hline & $\begin{array}{l}\text { C5. La calidad consiste en satisfacer las } \\
\text { expectativas de los alumnos y de las } \\
\text { organizaciones donde se colocan los } \\
\text { egresados. }\end{array}$ & $35 \%$ & $61 \%$ & $34 \%$ \\
\hline & $\begin{array}{l}\text { C3. La calidad consiste en el } \\
\text { cumplimiento de los objetivos y la } \\
\text { misión que ha establecido la institución. }\end{array}$ & $38 \%$ & $70 \%$ & $54 \%$ \\
\hline $\begin{array}{l}\text { Excelencia y } \\
\text { estándares }\end{array}$ & $\begin{array}{l}\text { C6. La calidad consiste en el } \\
\text { cumplimiento y la mejora de } \\
\text { estándares establecidos. }\end{array}$ & $39 \%$ & $69 \%$ & $54 \%$ \\
\hline $\begin{array}{l}\text { "Valor por } \\
\text { dinero" } \\
\text { (eficiencia) }\end{array}$ & $\begin{array}{l}\text { C7. La calidad consiste en conseguir } \\
\text { la eficiencia: obtener los mejores } \\
\text { resultados al menor costo. }\end{array}$ & $21 \%$ & $50 \%$ & $21 \%$ \\
\hline
\end{tabular}

Fuente: Cardona [Coord.] (en prensa).

Mijangos et al (2011, p. 27-30) han propuesto que las nociones de calidad pueden disponerse en un plano conformado por dos dimensiones que reflejan, 
respectivamente, el grado en que cada concepto de calidad se forma de acuerdo con un referente más o menos ajeno a la propia institución universitaria y el grado en que cada concepto representa una visión moderna o, por el contrario, tradicional, de la gestión de la calidad (ver figura 1).

Para Mijangos et al (2011) los conceptos de calidad representan el debate entre posturas contrapuestas. Por un lado, la interesada en poner en marcha un proceso de renovación en las instituciones universitarias por la vía de la introducción de la gestión de la calidad y los expedientes de rendición de cuentas. Para quienes defienden esta idea la solución sólo puede venir a través de la influencia de los valores ajenos a la universidad $\mathrm{y}$, en última instancia, de la puesta en marcha de sistemas de control externo, incluyendo los que aseguran el uso eficiente de los recursos públicos en el sector universitario. Lógicamente estas ideas se reflejan mejor en aquellas nociones de calidad basadas en referentes ajenos a la comunidad universitaria. En el lado contrario se sitúan quienes defienden los valores académicos y piensan que la autonomía universitaria es el mejor modo de garantizar su supervivencia. Sus ideas comulgan más fácilmente con las nociones de calidad inspiradas en las propias instituciones universitarias.

\section{Figura 1 - Adhesión de los docentes universitarios mexicanos a los conceptos} de calidad (Harvey y Green, 1993) ordenados en un espacio bidimensional. Frecuencias relativas de respuestas "alto" y "muy alto"*. México.

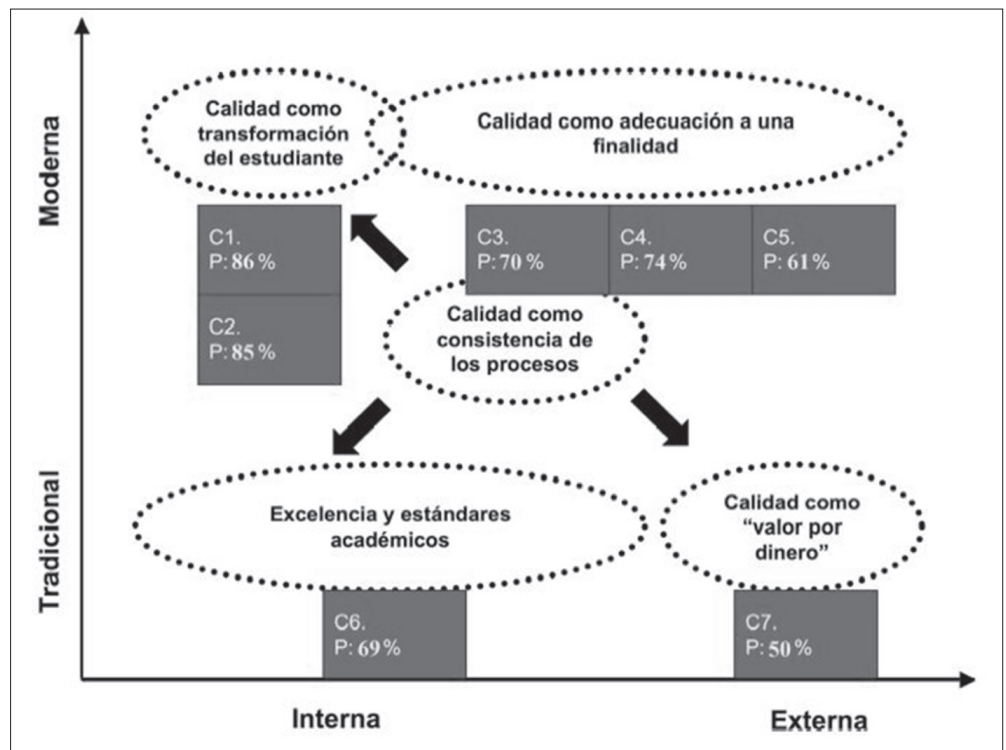

Fuente: a) de la estructura bidimensional: Mijangos el al (2011, p. 30); b) de los datos: Marúm et al (2011). 
Cuando se consulta a los académicos es corriente hallar éstos tienden a adherirse en mayor número a los conceptos de calidad modernos y basados en referentes internos a la comunidad académica. Así ocurre por ejemplo en España (MIJANGOS et al, 2011) o en México (MARÚM et al, 2011) (figura 1).

Estos datos no constituyen evidencia suficiente del rechazo unánime de los docentes hacia los programas de calidad. Al contrario, es claro que algunos de los principios de tales programas, como la identificación de los clientes o grupos de interés y su seguimiento, la elucidación de una misión con la que la institución se compromete, o la gestión eficiente de recursos, son admitidos por buena parte de los docentes. Lo que los datos demuestran es que los académicos no confían tan mayoritariamente en dichos valores como en los que consideran naturales de la comunidad académica. Para hallar evidencia de un verdadero conflicto de ideas en torno a la calidad y su gestión es preciso adoptar otro enfoque; cualquiera de estas dos vías es válida: a) comparar las opiniones del común de los docentes con las de aquellos que tienen responsabilidades de dirección, en particular en aquellos niveles de la administración universitaria en los que se ponen en marcha programas de calidad; b) solicitar a los docentes, su percepción de lo que realmente interesa a las instituciones para las que trabajan.

En el Reino Unido Lomas pidió a una muestra de administradores de centros universitarios que se decidieran por uno de entre cuatro conceptos de calidad (los cinco de Harvey y Green excepto el perfección o consistencia). E1 33\% de los encuestados prefirió la definición de calidad a partir de la adecuación a una finalidad previamente establecida; la idea de la transformación del estudiante alcanzó el segundo lugar en el recuento de votos. Si se comparan estos datos con los relativos a las opiniones de los académicos en general da la sensación de que las responsabilidades de administración provocan un cambio de opinión en los académicos y les obligan a preocuparse más por los objetivos establecidos y por satisfacer las expectativas de los stakeholders, y menos -relativamentepor el ideal educativo de transformar a los estudiantes.

Una evidencia más definitiva sobre las diferencias de opinión entre administradores y docentes se obtiene de los trabajos de López y Colombo [Coords.] (2011) y Cardona [Coord.] (2011). En ambos trabajos las definiciones operativas de calidad y los métodos empleados son idénticos para ambos grupos (administradores y docentes), si bien es cierto que la población investigada en cada caso es diferente: en el primer trabajo se encuestó a una muestra de decanos y directores de centros universitarios que imparten enseñanzas en el área de las ciencias sociales y jurídicas; mientras que en el segundo se trabajó con toda la población de docentes con independencia del tipo de centro o del área de 
conocimiento al que estuviesen adscritos. En cualquier caso, las opiniones de los docentes parecen ser bastante independientes del área de conocimiento y en centro de actividad (MIJANGOS et al, 2011), de manera que una comparación en estos términos, no siendo definitiva, sí parece razonable.

Tabla 3 - Adhesión de decanos y directores de centros, y de docentes a los diferentes conceptos de calidad en México*.

\begin{tabular}{|c|c|c|c|}
\hline $\begin{array}{c}\text { Concepto (Harvey } \\
\text { y Green, 1993) }\end{array}$ & Conceptos operativo & $\begin{array}{l}\text { Decanos y } \\
\text { directores de } \\
\text { centros }\end{array}$ & Docentes \\
\hline Transformación & $\begin{array}{l}\text { C1. La calidad consiste en desarrollar las } \\
\text { capacidades del estudiante para } \\
\text { avanzar en su propia transformación. } \\
\text { C2. La calidad consiste en formar } \\
\text { estudiantes capaces de asumir un } \\
\text { compromiso social. }\end{array}$ & $76 \%$ & $86 \%$ \\
\hline \multirow[b]{2}{*}{ Adecuación a un fin } & $\begin{array}{l}\text { C3. La calidad consiste en el } \\
\text { cumplimiento de los objetivos y la } \\
\text { misión que ha establecido la institución. }\end{array}$ & $80 \%$ & $70 \%$ \\
\hline & $\begin{array}{l}\text { C4. La calidad consiste en satisfacer las } \\
\text { expectativas de todos los involucrados } \\
\text { (alumnos, profesores, instituciones, } \\
\text { sociedad). } \\
\text { C5. La calidad consiste en satisfacer las } \\
\text { expectativas de los alumnos y de las } \\
\text { organizaciones donde se colocan los } \\
\text { egresados. }\end{array}$ & $69 \%$ & $61 \%$ \\
\hline $\begin{array}{l}\text { Excelencia y } \\
\text { estándares }\end{array}$ & $\begin{array}{l}\text { C6. La calidad consiste en el } \\
\text { cumplimiento y la mejora de estándares } \\
\text { establecidos. }\end{array}$ & $74 \%$ & $69 \%$ \\
\hline $\begin{array}{l}\text { "Valor por dinero" } \\
\text { (eficiencia) }\end{array}$ & $\begin{array}{l}\text { C7. La calidad consiste en conseguir la } \\
\text { eficiencia: obtener los mejores } \\
\text { resultados al menor costo. }\end{array}$ & $60 \%$ & $50 \%$ \\
\hline
\end{tabular}

* Los valores de la tabla expresan la frecuencia relativa de respuestas alto y muy alto a la pregunta sobre el acuerdo con cada una de las siete afirmaciones.

Fuente: Sobre decanos y directores: Marúm et al (2011a). Sobre docentes: Marúm et al (2009).

En México se aprecian al menos dos diferencias entre las opiniones de administradores y docentes (tabla 3). En primer lugar, los administradores muestran una mayor propensión a adherirse a la definición de calidad a partir de la misión de la institución, mientras que ocurre lo contrario con la idea de calidad como eficacia transformadora. En segundo lugar, se aprecia que las adhesiones de los administradores son más equilibradas que las de los docentes. Similares conclusiones se extraen de la evidencia reunida en Argentina y España. 
La segunda vía para contrastar si realmente existe un debate en el seno de las instituciones universitarias se basa en las percepciones que los docentes tienen sobre los objetivos que buscan sus instituciones, en contraste con lo que ellos mismos consideran más importante. También aquí puede recurrirse a los trabajos de Watty $(2006,2005)$, realizados sobre profesores australianos de contabilidad; Olaskoaga (coord.) (2009), sobre docentes de economía en cuatro países iberoamericanos; y Cardona (coord.) (2011), sobre docentes universitarios en Argentina, España y México. Los resultados de estos trabajos empíricos son coincidentes al menos en un aspecto: en todos ellos se concluye que los docentes perciben que las instituciones están bastante menos preocupadas que ellos mismos por lograr los objetivos de transformación de los estudiantes y excelencia de los estándares académicos (WATTY, 2005, p. 126). En un sentido similar, Marúm et al (2011) obtienen al menos dos conclusiones: a) Los docentes creen estar más concernidos por cualquier concepto de calidad que sus instituciones, con la excepción del concepto de calidad en el sentido de eficiencia económica; b) Los docentes muestran una marcada preferencia por los dos conceptos operativos asociados a la idea de calidad como transformación, al tiempo que atribuyen una preocupación mucho menor por estas dos cuestiones a las instituciones para las que trabajan.

\section{Valoración de la introducción de prácticas e instrumentos de gestión de la calidad en la enseñanza universitaria}

Descrito, aunque no resuelto, el debate sobre la noción de calidad en la enseñanza universitaria, sigue a continuación una pregunta de elevado interés tanto para los estudiosos de la calidad como para los responsables de la política universitaria: ¿cómo han aceptado los académicos la aplicación de programas de gestión de la calidad en sus instituciones?

El primero de los trabajos seleccionados que aborda esta cuestión es el de Elmuty et al (1996). Para sus autores existe evidencia de que la aplicación del Total Quality Management (TQM) en las instituciones universitarias del medio Oeste norteamericano cuenta con el apoyo de los académicos y de los más importantes stakeholders universitarios, aunque se admite que existe casi un tercio de escépticos. La pura aritmética -un $62 \%$ de partidarios frente a un tercio reticente- lleva a los autores a concluir que TQM permite a las universidades "atender mejor las demandas de sus clientes" (p. 41).

Sin embargo, observados con perspectiva, los datos de este trabajo pueden interpretarse de un modo distinto. En primer lugar, al valorar las respuestas de los entrevistados, los autores no consideraron, salvo en unos pocos casos, la 
posición que ocupan en la universidad. Las entrevistas se hicieron a 16 personas con responsabilidades administrativas y a otros 16 individuos que representaban a los diferentes stakeholders: docentes, estudiantes y empleadores. El texto del artículo no aclara el peso de cada uno de estos grupos en el total, pero si la muestra se diseñó de manera equilibrada no debía de haber en ella más de cinco o seis docentes, de los cuales cuatro formaban parte del grupo de críticos con TQM. Además, seis de entre los 16 administradores entrevistados se expresaron claramente en contra de TQM. Vistos así, los resultados no parecen demostrar que TQM cuente con un respaldo masivo por parte de los académicos. En todo caso, lo que sí evidencian los datos recopilados por Elmuty et al (1996) es que existe una clara discrepancia entre quienes trabajan en las organizaciones universitarias -en particular los docentes- y el resto de quienes mantienen alguna relación con ellas.

Interesa también revisar el modo en que se operacionalizan los principios y herramientas de TQM en este trabajo. Los principios que se atribuyen a TQM comprenden una serie de recomendaciones y mensajes habituales en el lenguaje del managemen: "adoptar un enfoque de cliente", "dotar de claridad y coherencia a los objetivos", o "establecer premios y reconocimientos ligados a la consecución de objetivos". Los efectos se distribuyen en cinco categorías: satisfacción, productividad, calidad, adaptabilidad y eficacia. Nadie duda de que estas categorías recogen aspectos importantes para cualquier organización; sin embargo, ninguna de ellas representa adecuadamente la esencia del trabajo universitario.

Por estas y algunas otras razones, las conclusiones del trabajo de Elmuty et al en el sentido de que TQM goza de un aceptable nivel de apoyo en las instituciones universitarias deben, como poco, someterse a disposiciones cautelares.

El trabajo de Newton $(1999,2001)$ representa un interesante hito en la literatura sobre opiniones de los docentes universitarios en torno a la calidad y su gestión. El contexto en el que se enmarca es el del sector británico de la educación superior, en una época -la década de los noventa- en la que se está poniendo en marcha un sistema nacional, con su correspondiente entramado institucional, para la evaluación externa de las instituciones de educación superior. Según el autor, los sistemas de garantía de calidad que en este contexto se pusieron en marcha en NewColl -la institución investigada- demostraron ser enteramente satisfactorios para los evaluadores externos, pero no lo fueron tanto para los académicos.

Newton pretendía demostrar que existe una importante brecha entre el discurso oficial sobre la calidad y su gestión y la realidad de su aplicación. Para ello 
utilizó, entre otras fuentes, una encuesta en la que se solicitó a los académicos de NewColl una valoración del flamante sistema de garantía de la calidad. La encuesta permitió apreciar una valoración muy positiva de la medida en que el sistema de garantía de calidad había permitido satisfacer los requerimientos y estándares establecidos en las evaluaciones interna y externa. Más del $90 \%$ de los encuestados estuvieron de acuerdo (en diferentes grados ${ }^{4}$ ) con estas afirmaciones, incluyendo a los docentes sin responsabilidades gestoras. Sin embargo, éstos fueron más tibios a la hora de expresar su aprobación que los coordinadores de curso o que los académicos con responsabilidades de gestión. Además, los porcentajes de aprobación fueron sensiblemente menores cuando la afirmación se pregunto si el sistema de calidad había llevado a mejoras para los estudiantes y para la plantilla. Un $85 \%$ de los administradores estuvo de acuerdo en que el sistema de calidad contribuyó a la mejora de la experiencia de los estudiantes, mientras que sólo un $40 \%$ de los docentes hizo lo propio. Y lo mismo ocurrió con las opiniones sobre la mejora de la plantilla, aunque de manera aun más acusada ( $73 \%$ frente al 22\%).

Las diferencias de actitud entre académicos y administradores son un hecho reconocido en la literatura. McInnis (1998) encontró posturas abiertamente diferentes entre los administradores profesionales y la plantilla docente de las instituciones australianas de educación superior tanto en lo que se refiere a los estilos de trabajo, como en cuanto a los valores de los que participan estos dos grupos. La novedad aquí reside en que las diferencias se aprecian también dentro del grupo de académicos y en función de la asunción de competencias de gestión.

Más evidencia en el mismo sentido puede hallarse en Rosa et al (2006) que trabajaron con las opiniones de rectores de universidades portuguesas y las de los coordinadores de los informes de autoevaluación. En su opinión ambos grupos se mostraron excesivamente optimistas (ROSA et al, 2006, p. 157) con respecto a los beneficios del sistema de evaluación de la calidad puesto en marcha en Portugal a partir de 2003; no obstante, los rectores resultaron más conformes con el discurso de la calidad y prestaron mayor atención a las cuestiones relacionadas con los procesos de gestión, mientras que los coordinadores estaban más interesados por los procesos básicos del core business universitario: la enseñanza y la investigación.

Con respecto a estos trabajos, los de Newton aportan un valor adicional; el que les confiere la viveza de detalles con la que se dibujan las reacciones de los docentes ante la puesta en marcha del sistema de garantía de calidad en

$4 \quad$ En la encuesta se trabajó con una escala de seis términos, de los cuales tres expresaban acuerdo y tres desacuerdo. 
NewColl (NEWTON, 2001, p. 10 y ss.). En general, los docentes no entienden qué hay de beneficioso en dedicar una parte de su tiempo a rellenar informes de evidencias, o a justificar programas y planes de estudio que a veces suponen para ellos un incómodo corsé en el ejercicio de su docencia. Frente a estas nuevas obligaciones, las actitudes del profesorado van desde el rechazo intransigente hasta la completa colaboración, pasando por actitudes más estratégicas como la adaptación racional a las nuevas condiciones de trabajo o la actitud de "ir tirando", es decir, cumplir los requisitos formales al tiempo que se ignora el verdadero sentido de las reformas.

Para Newton, los docentes tienen la capacidad de interferir en la aplicación de las políticas de calidad hasta el punto de dejarlas sin efecto, aunque a veces se observan actitudes mucho más sumisas. Entre la conformidad con el discurso oficial y la beligerancia intransigente hay dos planteamientos que resultan de interés. El primero conduce a los docentes a adaptarse a las nuevas condiciones mientras tratan de sacar provecho de sus aspectos más positivos, lo que incluye aprovechar oportunidades para desplegar propuestas desarrolladas a partir de sus propios valores y preocupaciones. Algo así parece haber ocurrido con ocasión de la creación del Espacio Europeo de Educación Superior (EEES). Sus objetivos inmediatos eran favorecer la movilidad y empleabilidad de los estudiantes, pero en países como Portugal (VIEIRA, 2002) o España (Ministerio de Educación y Ciencia, 2006) la inercia del proceso se ha aprovechado para iniciar una profunda revisión de los métodos docentes. El segundo planteamiento deriva en una aplicación puramente ritual de los procedimientos establecidos en las políticas de la calidad. Parte de las respuestas que Newton obtiene en sus entrevistas revela una postura cínica por parte de los académicos, que interpretan los sistemas de aseguramiento de la calidad como una serie de nuevas reglas a las que es posible sacar provecho.

El cinismo no es privativo de los docentes. Cartwright (2007, p. 296) observa que los académicos con responsabilidades de gestión en los programas de calidad también son conscientes de que están tomando parte en un "juego" cuyas reglas se establecen fuera del ámbito académico.

Los planteamientos de Newton y Cartwright han hallado respuesta en un trabajo reciente de Kleijnen et al (2011), que llega a conclusiones similares a las de Elmuty et al (1996).

Kleijnen et al encuestaron a 266 académicos que trabajan en treinta departamentos de las Universidades de Ciencias Aplicadas de Holanda ${ }^{5}$, y encontraron

5 La muestra incluyó a docentes en departamentos de administración de empresas, diversas especialidades de ingeniería y tecnología, sanidad, educación, bienestar social y bellas artes. 
una razonable aceptación de las políticas puestas en marcha por dichas instituciones en materia de gestión de la calidad. En general, las opiniones de los encuestados tomaron valores en torno a los tres puntos en una escala entre el 1 y el 5. La gestión de la calidad se llevó un "aprobado alto" en aspectos como el fomento del pensamiento crítico entre el profesorado $(3,66)$; la influencia del personal académico en la calidad del programa $(3,37)$; el desarrollo profesional del profesorado $(3,54)$; los procesos innovadores $(3,40)$; y su capacidad para convertir a los departamentos universitarios en organizaciones que aprenden $(3,49)$.

Los autores de esta investigación destacan que la opiniones de los docentes son menos acordes cuando se les consulta sobre el efecto de la gestión de la calidad en el grado de control al que se ven sometidos. Este ítem obtuvo una puntuación media de 2,52 en la escala entre 1 y 5, una valoración similar a la que los docentes concedieron a la afirmación de que la gestión de la calidad impide a los profesionales llevar adelante su personal contribución a la calidad de la educación $(2,57)$. Los autores de la investigación concluyeron que la evidencia choca con la idea extendida de que los docentes perciben la gestión de la calidad como una intensificación del control burocrático.

Para los partidarios de la gestión de la calidad en el sector universitario, el trabajo de Kleijnen et al (2011) ofrece una interpretación bastante amable. Sin embargo, los valores medios otorgados por los docentes a las afirmaciones sobre el impacto de la gestión de la calidad en la mejora de la enseñanza no son suficientemente altas - los propios autores los califican de "neutrales"- para concluir que existe unanimidad entre los docentes; al contrario, los resultados de Kleijnen et al son perfectamente compatibles con la existencia de posturas críticas entre los académicos.

\section{Opiniones de los docentes sobre sistemas de evaluación}

Toda la literatura sobre gestión de la calidad ha admitido el principio que establece la necesidad de evaluar las cosas para poder mejorarlas; de ahí el interés que ha mostrado siempre por la estandarización. Estandarización y evaluación son expedientes que pueden apoyarse recíprocamente: la evaluación es más fácil cuando se realiza contra un estándar establecido. Por su parte, evaluar sistemáticamente los resultados y rendimientos obtenidos permite modificar los propios estándares, adaptándolos a lo que la sociedad puede esperar del desarrollo de la economía y la tecnología en cada momento.

La historia de las instituciones universitarias no es ajena a los estándares ni a la evaluación, lo que ocurre es que lo habitual es que los estándares se 
establezcan sobre los inputs más relevantes de la actividad educativa -los docentes y los estudiantes- pero no sobre los procedimientos o los resultados. Es tradicional que las universidades evalúen a los docentes antes de contratarlos, y también que establezcan algún método de selección del alumnado. La mayor parte de la responsabilidad del control de la calidad en las universidades se deposita en el reclutamiento de los docentes. Y debe ser así, porque una vez se ha comprobado que el académico cumple con determinados estándares de aptitud científico-técnica y de compromiso, se le otorga una considerable autonomía para que juzgue las circunstancias, diseñe y planifique sus tareas, y evalúe a sus estudiantes.

La gestión de la calidad ha enfatizado los expedientes de estandarización y evaluación de procedimientos y resultados. Por ejemplo, la evaluación externa de los títulos y programas universitarios obliga a los docentes a documentar extensamente las planificación que sus actividades docentes, incluyendo la identificación de las competencias que se trabajarán en los cursos o el establecimiento de sistemas de evaluación del alumno.

Por su parte, los sistemas de garantía de la calidad las universidades, implican consultas a los estudiantes, antiguos alumnos y empleadores. sobre la calidad de los resultados del proceso de enseñanza.

La cuestión ahora consiste en conocer la hospitalidad con la que los académicos han acogido estas nuevas prácticas.

La evidencia empírica acumulada describe la reacción de los docentes en términos de hostilidad. En este sentido es preciso valorar varios aspectos: a) las consecuencias en el trabajo de los académicos; b) la eficacia que los académicos les atribuyen, y c) las instancias responsables y los medios empleados para la evaluación.

El trabajo de Newton $(1999,2001)$ es el más expresivo de lo escasamente receptivos que se han mostrado los académicos con los sistemas de evaluación. En él se recogen reflexiones sobre el efecto de los sistemas de calidad sobre la autonomía del académico: "Los sistemas [se refiere a los sistemas de evaluación y garantía de la calidad] tienen una lógica inmanente tal que hace que las experiencias que he tenido con ellos resulten opresivas ... porque inhiben la autonomía que me corresponde ... como profesional".

Otra de las razones del reside en el tiempo que consumen unos procedimientos de evaluación que habitualmente obligan a redactar informes y requieren una extensiva documentación. La referencia a la carga de trabajo adicional es una constante de las consultas a los docentes (URSIN et al, 2008, p. 116-117; ROSA et al, 2006, p. 153; NEWTON ,2001). 
Pero la sensación de carga de trabajo se debe en parte a la escasa eficacia que los académicos atribuyen a estos métodos. Docentes participantes en entrevistas consideraron que los informes generan un flujo de información hacia lo alto de las organizaciones que no termina de revertir en su trabajo, ni tampoco en la mejora de la enseñanza (NEWTON, 2001). Los métodos empleados para tampoco se consideran eficaces: los profesores prefieren el diálogo informal con los estudiantes que las encuestas tipo (NEWTON, 2001, p. 15), máxime cuando es extraño que los resultados de éstas sean objeto de análisis (URSIN et al, 2008, p. 114).

También en este campo las responsabilidades de gestión influyen en la percepción de los académicos. Por ejemplo, los rectores y coordinadores de programas de las universidades portuguesas entrevistados por Rosa et al (2006) valoraron positivamente el impacto del sistema de evaluación de la calidad en las universidades puesto en marcha en su país. Los rectores otorgaron puntuaciones entre los tres y los cuatro puntos (en una escala del uno al cinco) a aspectos como la oportunidad de las recomendaciones (3,2 puntos); la relevancia de las recomendaciones $(3,4)$; el grado de aplicación de las recomendaciones $(3,4)$ o la utilidad de las visitas externas para la evaluación de los programas $(3,9)$. Los propios autores de este trabajo señalan que las valoraciones fueron excesivamente optimistas (ROSA et al, 2006: 157); pero quizá es más relevante que el ítem que recibió mayor puntuación no tiene que ver el efecto de del sistema de evaluación en la calidad de la educación, sino con la utilidad de las visitas de los evaluadores de cara a la obtención de la acreditación.

Los trabajos en Cardona [coord.] (2011) se refieren específicamente a la evaluación de la actividad docente del profesor, y su novedad reside en que la influencia de estos métodos se evalúa junto con la de otra serie de factores (hasta un total de 26) que a priori se interpretan relevantes para la calidad de la enseñanza universitaria, así como en que la consulta especifica tres tipos de evaluación: evaluación mediante auto-informes del profesor o auto-evaluación, evaluación por parte de los responsables académicos, y evaluación a través de encuestas de opinión realizadas a los estudiantes. Los expedientes de evaluación tendieron a ocupar los últimos puestos en el orden de prelación establecido por los docentes encuestados (tabla 4).

Estos datos ofrecen una nueva perspectiva de la eficacia otorgada por los docentes a los expedientes de evaluación de su trabajo. Puede que los docentes reconozcan algún valor en estos instrumentos, pero queda claro que hay otros aspectos de la enseñanza que son más importantes y a los que las políticas universitarias deberían dirigir su mirada. 
Tabla 4 - Impacto en la calidad que los docentes atribuyen a tres diferentes métodos de evaluación de la actividad docente*.

\begin{tabular}{|c|c|c|c|c|c|c|}
\hline & \multicolumn{2}{|c|}{ España } & \multicolumn{2}{|c|}{ Argentina } & \multicolumn{2}{|c|}{ México } \\
\hline & FRA* $^{*}$ & Posición** & $\mathrm{FRA}^{*}$ & Posición** & FRA $^{*}$ & Posición** \\
\hline $\begin{array}{l}\text { Evaluación de la actividad } \\
\text { docente delprofesorado a través } \\
\text { de encuestas de opinión de los } \\
\text { alumnos. }\end{array}$ & $28 \%$ & 24 & $45 \%$ & 22 & $53 \%$ & 24 \\
\hline $\begin{array}{l}\text { Evaluación de la actividad } \\
\text { docente delprofesorado a través } \\
\text { de informes } \\
\text { elaborados por los responsables } \\
\text { académicos. }\end{array}$ & $21 \%$ & 25 & $40 \%$ & 24 & $45 \%$ & 25 \\
\hline $\begin{array}{l}\text { Evaluación de la actividad } \\
\text { docente del profesorado a } \\
\text { través de autoinformes. }\end{array}$ & $18 \%$ & 26 & $24 \%$ & 26 & $43 \%$ & 26 \\
\hline
\end{tabular}

\section{DISCUSIÓN Y CONCLUSIONES}

Resulta difícil conducir toda la evidencia acumulada hasta la fecha hacia una conclusión sencilla y definitiva sobre la manera en que los académicos valoran la introducción de los programas de calidad en las instituciones para las que trabajan; pero sí son pertinentes algunas reflexiones en torno a la evidencia recopilada en este artículo.

1. La primera conclusión se refiere a la propia evidencia que, además de fragmentaria y dispersa, se ha obtenido por aplicación de métodos diversos cuando no radicalmente diferentes entre sí. Por si esto fuera poco, cuando los métodos empleados se examinan con detenimiento se percibe que todos sufren algún tipo de vicio. Las investigaciones basadas en encuestas han conseguido tasas de respuesta bastante bajas, lo que puede haber sesgado los resultados. Los trabajos de corte más cualitativo, como el de Newton $(1999,2001)$, ofrecen por lo general descripciones más ricas en matices que los casi siempre previsibles resultados de las encuestas, pero quizá estén reflejando realidades muy particulares que no son extensibles a otras situaciones y contextos. Ocurre, además, que los resultados de la aplicación de diferentes metodologías no siempre son compatibles entre sí. 
2. Los contextos geográfico, temporal e institucional también son relevantes a la hora de valorar las respuestas de los académicos. No se puede esperar que los docentes valoren las contribuciones de la gestión de la calidad in abstracto; lo hacen teniendo en mente las prácticas con las que se encuentran en su contexto de trabajo. La influencia del momento también se percibe en las expectativas de los docentes sobre las novedades derivadas de la puesta en marcha de los créditos ECTS. Por ejemplo, García-García et al (2009) concluyen en su trabajo que los docentes españoles eran más optimistas que los italianos con respecto a las consecuencias del EEES y sugieren que ello pudo deberse a que en el momento en que se realizó la encuesta aún no habían tenido tiempo de conocer de primera mano ni la aplicación de los programas ni sus consecuencias. Por otro lado, sabemos del trabajo de Zbaracki (1998) que detrás de la gestión de la calidad también existe un discurso. Las investigaciones de Newton confirman que los propios académicos son conscientes del discurso oficial sobre la calidad y a veces se adhieren a él por pura conveniencia. Si éste es el caso, la propia difusión del discurso puede estar influyendo en las respuestas de los docentes.

3. A pesar de todo, es posible extraer algunas conclusiones definitivas. Por ejemplo, las opiniones de los académicos difieren en función de la posición que ocupan en la jerarquía de las instituciones para las que trabajan. Tal cosa se observa lo mismo en trabajos tan diferentes en metodología y enfoque como los de Newton $(1999,2001)$ y Elmuty et al (1996).

4. Aunque no existe evidencia suficiente al respecto, es razonable suponer que tales diferencias de opinión se deben a las diferentes concepciones de calidad de los académicos. La evidencia demuestra que el ejercicio de la función directiva representa una línea de fractura en este sentido. Se ha comprobado que los académicos en puestos de gestión ven el objetivo de la calidad de una manera diferente, están más dispuestos a aceptar las normas impuestas por los sistemas de aseguramiento de la calidad, y se muestran más optimistas con respecto a las consecuencias de los programas de calidad y su aseguramiento en las instituciones universitarias. También existen cuestiones en los que administradores y docentes coinciden. Por ejemplo, buena parte de los administradores consultados por Elmuty et al (1996) estaba de acuerdo en que la gestión de la calidad, tal y como convencionalmente se plantea, no se 
adecua a las especiales condiciones de las instituciones universitarias. Por su parte, Newton (2001) y Cartwright (2007) encontraron dosis similares de cinismo entre los académicos con y sin responsabilidades de gestión.

5. Sean cuales sean las razones de las diferencias de opinión entre y dentro de estas dos categorías de académicos, la literatura alerta sobre la posibilidad de lo que se ha denominado un "conflicto latente en las instituciones universitarias" (BARANDIARAN et al, 2011).

La existencia de grupos con opiniones diferentes e incluso opuestas con respecto a estas cuestiones no constituye el mejor escenario para una universidad que inevitablemente debe afrontar cambios en el futuro.

6. La cuestión de las opiniones de los docentes sobre la gestión de la calidad se ha planteado desde enfoques muy diferentes. Buena parte de los autores cuyo trabajo se ha valorado y analizado en este capítulo adoptan un enfoque puramente pragmático (en este grupo se encuentran Elmuty et al, 1996; o Fernández et al, 2010, por citar dos ejemplos). Para ellos las reacciones de los docentes críticos con el discurso de la calidad constituyen un mero obstáculo para la aplicación de unas reformas que a priori se consideran deseables. La cuestión práctica se reduce a encontrar el modo de salvar dicho obstáculo, de ahí que las explicaciones que proponen de las reacciones de los docentes tiendan a la simplicidad. El rechazo de los docentes se atribuye bien a la falta de información, o bien a algún tipo de comportamiento inveterado típico en las instituciones universitarias. Estos trabajos no están diseñados para comprender la posición de los docentes, ni para descubrir -si existe- cuál es la racionalidad de su rechazo. Newton (2001) es el primero en rebelarse contra este planteamiento. Para él es preciso interpretar las posturas de los académicos, sin descartar de entrada la oportunidad de sus críticas y planteamientos.

Los autores de este artículo nos hallamos más próximos a la propuesta de Newton que al enfoque pragmático, aunque admitimos la complementariedad de ambos planteamientos. En nuestra opinión la postura de los docentes se debe, al menos en parte, a la inercia de las soluciones estructurales de las organizaciones universitarias. La configuración estructural típica en las instituciones universitarias se conoce como burocracia profesional (MINTZBERG, 1985) y constituye una solución válida para organizaciones que desarrollan tareas 
complejas y difíciles de estandarizar. Esta configuración resulta muy favorable para los académicos, que gozan de una enorme autonomía en el ejercicio de su trabajo y disfrutan de unas relaciones de prevalencia con respecto al resto de los elementos estructurales de la organización. En estas condiciones es lógico que traten de preservar su posición. Desarrollar y contrastar esta hipótesis sería la continuación natural de la investigación recogida en este artículo.

\section{REFERENCIAS}

ARAYA, L.; ARIAS, K.; IBARRA, A. Percepción del profesorado universitario sobre los factores ligados a la calidad de la enseñanza: resultados de Chile. En: OLASKOAGA, J. (Coord.). Hacia una educación superior de calidad. Un análisis desde la perspectiva del profesorado en Argentina, Chile, España y México. La Plata: EDULP, 2009.

BARANDIARAN, M. et al. Conclusiones de un análisis comparado: nociones de calidad universitaria en Iberoamérica. En: LÓPEZ ARMENGOL, M.; COLOMBO, M. P. (Coords.). Hacia una educación superior de calidad. Una mirada de quienes gestionan las universidades en Argentina, España y México. La Plata: EDULP, 2011. v. II.

BARRENETXEA, M. et al. Percepción del profesorado universitario sobre los factores ligados a la calidad de la enseñanza: resultados de España. En: OLASKOAGA, J. (Coord.). Hacia una educación superior de calidad. Un análisis desde la perspectiva del profesorado en Argentina, Chile, España y México. La Plata: EDULP, 2009.

BOADEN, R. What is total quality management ... and does it matter? Total Quality Management \& Business Excellence, United Kingdom, v. 8, n. 4, p. 153-171, 1997.

CARDONA, A. (Coord.). Calidad en la educación superior: ¿qué modelo y en qué condiciones? La opinión del profesorado en Argentina, España y México. Guadalajara, México: Servicio Editorial de la Universidad de Guadalajara, 2012. (En prensa)

CARDONA, A. et al. Concepto y Determinantes de la Calidad de la Educación Superior. Un Sondeo de Opinión entre Profesores de Universidades Españolas. Archivos Analíticos de Políticas Educativas, Tempe, AZ, v. 17, n. 10, 2009. 
CARTWRIGHT, M. J. The rhetoric and reality of "quality" in higher education". Quality Assurance in Education, United Kingdom, v. 15, n. 3, p. 287-301, 2007.

ELMUTY, D.; KATHAWALA, Y.; MANIPPALLILI, M. Are total quality management programs worth the effort? International Journal of Quality \& Reliability Management, United Kingdom ,v. 13, n. 6, p. 29-42, 1996.

FERNÁNDEZ M. J.; CARBALLO, R.; GALÁN, A. Faculty attitudes and training needs to respond the new European Higher Education challenges. Higher Education, n. 60, p. 101-118, 2010.

FERRER, F. et al. N. Las opiniones y actitudes del profesorado universitario delante el Espacio Europeo de Educación Superior: propuestas para la implementación del sistema de créditos europeos (ECTS). Barcelona: Universidad Autónoma de Barcelona. Ministerio de Educación y Ciencia, 2004.

GARCÍA-GARCÍA, M. J.; GONZÁLEZ, C.; ARGÜELLES, R. Methodological changes in technical teaching in order to the European higher education area comparison between countries: Italy and Spain. Procedia. Social and Behavioral Sciences, n. 1, p. 2701-2706, 2009.

HARVEY, L.; GREEN, D. Defining Quality. Assessment \& Evaluation in Higher_Education, United Kingdom v. 18, p. 1, p. 9-34, 1993.

KLEIJNEN, J. et al. Does internal quality management contribute to more control or to improvement of higher education? A survey on faculty's perceptions. Quality Assurance in Education, United Kingdom, v. 19, n. 2, p. 141-155, 2011.

LOMAS, L. Does the development of mass education necessarily mean the end of quality? Quality in Higher Education, United Kingdom, v. 8, p. 1, p. 71-79, 2002.

LOMAS, L. Are Students Customers? Perceptions of Academic Staff. Quality in Higher Education, United Kingdom, v. 13, n. 1, p. 31-44, 2007.

LÓPEZ ARMENGOL, M.; COLOMBO, M. P. (Coords.). Hacia una educación superior de calidad. Una mirada de quienes gestionan las universidades en Argentina, España y México. La Plata: EDULP, 2011. v. II 
MARÚM, E. et al. Percepción del profesorado universitario sobre los factores ligados a la calidad de la enseñanza: resultados de México. En: OLASKOAGA, J. (Coord.). Hacia una educación superior de calidad. Un análisis desde la perspectiva del profesorado en Argentina, Chile, España y México. La Plata :EDULP, 2009.

MARÚM, E. et al. Opiniones sobre la calidad de la enseñanza universitaria de decanos y directores de centros universitarios de ciencias sociales y jurídicas. En: LÓPEZ ARMENGOL, M.; COLOMBO, P. Hacia una educación superior de calidad. Una mirada de quienes gestionan las universidades en Argentina, España y México. La Plata: EDULP, 2011a. v. II MARÚM, M. et al. El profesorado universitario mexicano ante la calidad de la educación superior: factores determinantes. En: CARDONA (Coord.). Calidad en la educación superior: ¿qué modelo y en qué condiciones? La opinión del profesorado en Argentina, España y México. Guadalajara, Mexico: Servicio Editorial de la Universidad de Guadalajara, 2012. (En prensa).

MCINNIS, C. Academics and Professional Administrators in Australian Universities: dissolving boundaries and new tensions. Journal of Higher Education Policy and Management, Sidney, v. 20, n. 2, p. 161-173, 1998.

MIJANGOS, J.J. et al. Enseñanza universitaria de calidad: profesorado, alumnado e institución. Bilbao: Servicio editorial de la Universidad del País Vasco, 2011.

MIJANGOS, J.J. et al El profesorado universitario español ante la calidad de la educación superior: factores determinantes. En: CARDONA, A. (Coord.). Calidad en la educación superior: ¿qué modelo y en qué condiciones? La opinión del profesorado en Argentina, España y México. Guadalajara, Mexico: Servicio Editorial de la Universidad de Guadalajara, 2011.

MINTZBERG, H. La estructuración de las organizaciones. Madrid: Alianza, 1985.

NEWTON, J. An evaluation of the impact of external quality monitoring on a Higher Education College (1993-98). Assessment \& Evaluation in Higher Education, United Kingdom, v. 24, n. 2, 1999.

NEWTON, J. Views from below: academics coping with quality. paper Presentado en el sexto seminario QHE, en asociación con EAIR; SRHE. Birmingham, 26 de mayo de 2001. 
OLASKOAGA, J. (Coord.). Hacia una educación superior de calidad. Un análisis desde la perspectiva del profesorado en Argentina, Chile, España y México. La Plata: EDULP, 2009.

PERSOGLIA, L.; LÓPEZ ARMENGOL, M.; ZOLOA J.I. Percepción del profesorado universitario sobre los factores ligados a la calidad de la enseñanza: resultados de la Argentina. En: OLASKOAGA, J. (Coord.). Hacia una educación superior de calidad. Un análisis desde la perspectiva del profesorado en Argentina, Chile, España y México. La Plata: EDULP, 2009 .

ROSA, M. J.; TAVARES, D.; AMARAL, A. Institutional consequences of quality assessment. Quality in Higher Education, United Kingdom, v. 12, n. 2, p. 145-159, 2006.

URSIN, J. et al. Evaluation and Quality Assurance in Finnish and Italian Universities in the Bologna Process. Quality in Higher Education, United Kingdom, v. 14, n. 2, p. 109-120, 2008.

VEIGA, A.; AMARAL, A. Survey on the implementation of the Bologna process in Portugal. Higher Education, New York, NY, v. 57, n. 1, p. 57-69, 2009.

VIEIRA, F. Pedagogic Quality at University: What teachers and students think. Quality in Higher Education, United Kingdom, v. 8, n. 3, p. 255 272, 2002.

WATTY, K. Quality in accounting education: what say the academics? Quality Assurance in Education, United Kingdom, v. 13, n. 2, p. 120-131, 2005.

WATTY, K. Want to know about quality in education? Ask an academic. Quality in Higher Education, United Kingdom, v. 12, n. 3, p. 291-301, 2006.

ZBARACKI, Mark. The rhetoric and reality of total quality management. Administrative Science Quarterly, Ithaca, NY, sept. 1998. 
